\title{
Theoretical Studies of Tunnel Magnetoresistance and Shot Noise in a Schottky-Barrier Carbon Nanotube Transistor with Ferromagnetic Contacts
}

\author{
S. KROMPIEWSKI \\ Institute of Molecular Physics, Polish Academy of Sciences \\ M. Smoluchowskiego 17, 60-179 Poznań, Poland \\ A Schottky-barrier carbon nanotube field-effect transistor with ferro- \\ magnetic contacts was modelled. The theoretical method combines a tight- \\ binding model and the non-equilibrium Green function technique. Tunnel \\ magnetoresistance as well as current noise of the carbon nanotube field- \\ effect transistor are the main issues addressed in this study. It is shown \\ that the former may exceed $50 \%$, whereas the latter is characterized by the \\ Poissonian Fano factor $(F)$ within the sub-threshold region, and the sub- \\ Poissonian $F \approx 0.5$ for elevated gate voltages. Remarkably, reorientation \\ of relative magnetization alignments of the contacts may lead to noticeable \\ changes in the current noise.
}

PACS numbers: 85.75.Hh, 75.47.De, 42.50.Lc, 73.63.Fg

\section{Introduction}

In search for alternative technologies to the conventional one based on silicon, there have recently been intensive studies on new methods based on nanostructured materials which are formed by self-organized bottom-up approaches rather than the lithographical patterning. Along with various molecular candidates $[1,2]$, most promising seem to be carbon nanotubes $[3,4]$. Here, a simple theoretical approach has been developed that elucidates the performance of Schottky-barrier nanotube transistor with ferromagnetic contacts. Energy band diagrams for a semiconducting single wall carbon nanotube (SWCNT) are self-consistently determined to visualize the Schottky-barrier evolution as a function of bias and gate-voltages. The emphasis is put on analyzing current gate-voltage characteristics as well as shot (and thermal) noise [5].

\section{Formalism and results}

A device, modelled here, is composed of a zigzag CNT end-contacted to $s$-electron metal contacts of fcc (111) geometry. The present model adapts basic 
ideas of Refs. [6-8] developed for conventional Si-based transistors) to the case of CNT Schottky-barrier field-effect transistor (FET) with ferromagnetic contacts. This approach combines a simplified (analytical) treatment of the electrostatics, and the state-of-the-art non-equilibrium Green function (GF) technique.

As shown in Ref. [6], a solution to the one-dimensional Poisson equation reads

$$
\begin{aligned}
V(x) & =\int \mathrm{d} x^{\prime} v\left(x, x^{\prime}\right)\left(\frac{\rho\left(x^{\prime}\right)}{\epsilon_{0} \epsilon_{\mathrm{CNT}} A}+\frac{V_{\mathrm{G}}}{\lambda^{2}}\right) \\
& +\left(V_{\mathrm{S}} \sinh \frac{L-x}{\lambda}+V_{\mathrm{D}} \sinh \frac{x}{\lambda}\right) / \sinh \frac{L}{\lambda},
\end{aligned}
$$

where $v\left(x, x^{\prime}\right)=\frac{\lambda}{2}\left[\mathrm{e}^{-\left|x-x^{\prime}\right| / \lambda}-\mathrm{e}^{-\left(x+x^{\prime}\right) / \lambda}+\mathrm{e}^{L / \lambda}\left(\cosh \frac{x-x^{\prime}}{\lambda}-\cosh \frac{x+x^{\prime}}{\lambda}\right) / \sinh \frac{L}{\lambda}\right]$. Above, $\lambda=(R / 2) \sqrt{2\left(\epsilon_{\mathrm{CNT}} / \epsilon_{0 x}\right) \ln \left(1+d_{0 x} / R\right)+1}$ is the effective screening length [8]; $\epsilon_{\mathrm{CNT}}, R, L$ and $A$ stand for the dielectric constant, radius, length and cross-sectional area of the CNT, whereas $\epsilon_{0 x}$ and $d_{0 x}$ are the dielectric constant of the coaxial $\mathrm{SiO}_{2}$-gate layer and its thickness. Furthermore: $V_{\mathrm{S}}, V_{\mathrm{D}}, V_{\mathrm{G}}$ are source, drain, and gate voltages and $f_{\mathrm{S}, \mathrm{D}}$ are the Fermi functions at source and drain potentials $\left(V_{\mathrm{S}}\right.$ and $\left.V_{\mathrm{D}}\right)$.

Having got the potential profile $V(x)$, the current and zero-frequency noise power can be expressed in terms of non-equilibrium GFs $G^{\mathrm{r}}$ and $G^{\mathrm{a}}=\left(G^{\mathrm{r}}\right)^{\dagger}$ from

$$
\begin{aligned}
I= & \frac{e}{h} \int \mathrm{d} E\left(f_{\mathrm{S}}-f_{\mathrm{D}}\right) \operatorname{Tr}[T(E)], \\
S= & \frac{2 e^{2}}{h} \int \mathrm{d} E\left\{\left[f_{\mathrm{S}}\left(1-f_{\mathrm{S}}\right)+f_{\mathrm{D}}\left(1-f_{\mathrm{D}}\right)\right] \operatorname{Tr}[T(E)]\right. \\
& \left.+\left(f_{\mathrm{S}}-f_{P} \mathrm{D}\right)^{2} \operatorname{Tr}[T(E)(1-T(E))]\right\},
\end{aligned}
$$

where the transmission matrix $T=-\left(\Sigma_{\mathrm{S}}^{\mathrm{r}}-\Sigma_{\mathrm{S}}^{\mathrm{a}}\right) G^{\mathrm{r}}\left(\Sigma_{\mathrm{D}}^{\mathrm{r}}-\Sigma_{\mathrm{D}}^{\mathrm{a}}\right) G^{\mathrm{a}}$. The energydependent self-energies are $\Sigma_{\alpha}(E)=v_{\mathrm{c}} g_{\alpha}(E) v_{\mathrm{c}}^{\dagger}$ with contact surface GFs $(g)$ and the contact/CNT coupling matrices $\left(v_{\mathrm{c}}\right)[9,10]$.

The density matrix can then be calculated from $\hat{\rho}=\frac{1}{2 \pi} \int \mathrm{d} E\left[\mathrm{i} f_{\mathrm{D}}\left(G^{\mathrm{r}}-G^{\mathrm{a}}\right)+\right.$ $\left.\left(f_{\mathrm{S}}-f_{\mathrm{D}}\right) G^{\mathrm{r}} \Gamma_{\mathrm{S}} G^{\mathrm{a}}\right]$. Obviously, the procedure is self-consistent because the potential file $\mathrm{V}(\mathrm{x})$ (Eq. (1)) enters the GFs. It should also be stressed that the self-energies, $\Sigma$, are spin-dependent — and so are the currents, the noises and the carrier densities — due to spin polarization of the contacts (set to $50 \%$ ).

The quantities of special interest here which will be analyzed in more detail in the following are the tunnel magnetoresistance (TMR) and the Fano factor (F) defined as: $\mathrm{TMR}=\left(\mathrm{I}_{\mathrm{P}}-I_{\mathrm{AP}}\right) / I_{\mathrm{P}}$ and $F=\left(S^{\uparrow}+S^{\downarrow}\right) /\left(2 e\left(I^{\uparrow}+I^{\downarrow}\right)\right)$, where $I_{\mathrm{P}}\left(I_{\mathrm{AP}}\right)$ is the current for the parallel, $\mathrm{P}$ (antiparallel, AP) alignment of the magnetizations of the contacts. The GF's non-equilibrium formalism, adopted here, is not the most general one. Still, it is valid for the present room temperature model, where electron correlations are described on the mean-field level. At low 
temperatures, within either Coulomb blockade or the Kondo regimes, Eqs. (2) would need improving $[11,12]$.
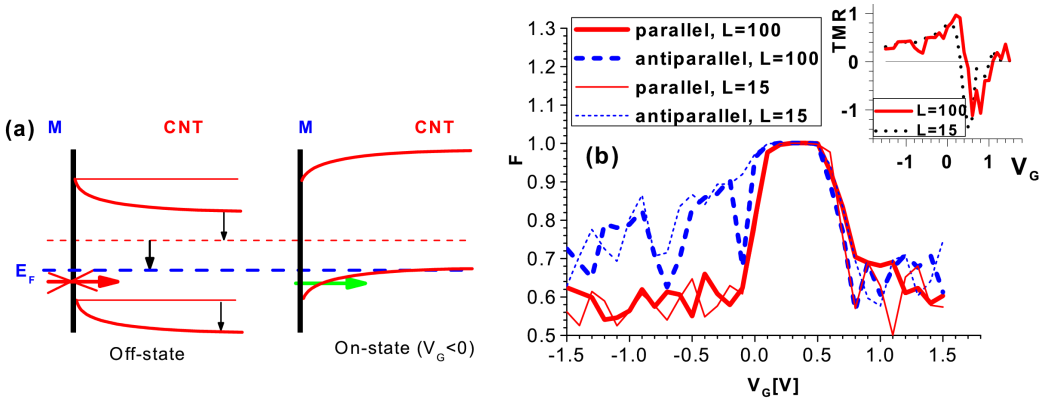

Fig. 1. (a) Schematic of the band diagram. The thin lines show the band gap of the isolated CNT, whereas the thick ones illustrate the band bending (Fermi level line-up). In the on-state the holes can tunnel through the Schottky barrier. (b) The Fano factor for the P- and AP-alignments vs. gate-voltage (solid and dashed lines, respectively). The CNT lengths are $L=100 \mathrm{uc}$ (thick lines) and $L=15 \mathrm{uc}$ (thinner lines). Drain/source voltage is equal to $0.2 \mathrm{~V}$ and temperature $T=300^{\circ} \mathrm{K}$. Inset: The TMR dependence on $V_{\mathrm{G}}$ for $L=100$ (solid line) and $L=15$ (dotted line).

The FET under consideration here is based on a zigzag single wall CNT with a chiral vector $(14,0)$ (the diameter close to $1 \mathrm{~nm}$ and the energy gap $0.7 \mathrm{eV}$ ), cylindrically gated with a $2.5 \mathrm{~nm}$ thick insulator. It is assumed that the work function (WF) of the metal contacts is greater than that of the CNT. Consequently the metal Fermi function lies below the mid-gap of the CNT, as shown in Fig. 1a. The corresponding Fano factors and TMR coefficients are shown in Fig. 1b as functions of the gate voltage. The solid line in the inset to Fig. 1b denotes TMR for the longer tube of $L=100$ unit cells (uc), whereas the dotted line is for $L=15$ uc. As expected, for the quasi ballistic limit considered here, the length effect is rather minor (and merely quantitative).

\section{Conclusions}

Combining a simplified treatment of the Poisson equation with the state-ofthe-art non-equilibrium GF technique, a model Schottky-barrier CNT-based FET is proposed. Energy band diagrams for a semiconducting single wall carbon nanotube are self-consistently determined to visualize the Schottky-barrier evolution as a function of bias and gate voltages. As regards the impact of ferromagnetic contacts, although the Schottky-barrier is practically the same for the parallel and antiparallel alignments (at fixed bias and gate voltages), the corresponding currents and shot noises are definitely different, due to different spin-dependent surface densities of states of the contacts. Remarkably, it turns out that reorientation of the respective alignment of the magnetic contacts from the antiparallel to 
the parallel configuration results in noticeable improvement of the noise-to-current ratio.

\section{Acknowledgments}

This work was suported by the EU FP6 grants: CARDEQ under contract No. IST-021285-2, and SPINTRA under contract No. ERAS-CT-2003-980409. We acknowledge the Poznań Supercomputing and Networking Center for the computing time.

\section{References}

[1] Z.H. Xiong, D. Wu, Z. Valy Vardeny, J. Shi, Nature 427, 821 (2004).

[2] W.J.M. Naber, S. Faez, W.G. van der Wiel, cond-mat/0703455v1.

[3] R. Saito, M.S. Dresselhaus, G. Dresselhaus, Physical Properties of Carbon Nanotubes Imperial College Press, London 1998.

[4] A.P. Graham, G.S. Duesberg, W. Hoenlein, F. Kreupl, M. Liebau, R. Martin, B. Rajasekharan, W. Pamler, R. Seidel, W. Steinhoegl, E. Unger, Appl. Phys. A 80, 1141 (2005).

[5] Ya.M. Blanter, M. Büttiker, Phys. Rep. 336, 1 (2000).

[6] K.M. Indlekofer, J. Knoch, J. Appenzeller, Phys. Rev. B 72, 125308 (2005).

[7] F.G. Pikus, K.K. Likharev, Appl. Phys. Lett. 71, 3661 (1997).

[8] P. Auth, J.D. Plummer, IEEE El. Dev. Lett. 18, 74 (1997).

[9] S. Krompiewski, J. Martinek, J. Barnaś, Phys. Rev. B 66, 073412 (2002).

[10] S. Krompiewski, Semicond. Sci. Technol. 21, S96 (2006).

[11] Antti-Pekka Jauho, J. Phys., Conf. Series 35, 313 (2006).

[12] R.K. Lake, R.R. Pandey, cond-mat/0607219. 